\title{
Parameter Estimation and Stress-Strength Model of Power Lomax Distribution: Classical Methods and Bayesian Estimation
}

\author{
Ehab M. Almetwally*1 and Hisham. M. Almongy ${ }^{2}$ \\ ${ }^{1}$ Faculty of Business Administration, Delta University of Science and Technology, Mansoura, Egypt \\ ${ }^{2}$ Faculty of Commerce, Mansoura University, Mansoura, Egypt
}

\begin{abstract}
In this paper, parameter estimation for the power Lomax distribution is studied with different methods as maximum likelihood, maximum product spacing, ordinary least squares, weighted least squares, Cramér-von Mises and Bayesian estimation by Markov chain Monte Carlo (MCMC). Robust estimation of the stress-strength model for the Power Lomax distribution is discussed. We propose that the method of maximum product of spacing for reliable estimation of stress-strength model as an alternative method to maximum likelihood and Bayesian estimation methods. A numerical study using real data and Monte Carlo Simulation is performed to compare between different methods.
\end{abstract}

Keywords Bayesian estimation; Cramér-von Mises; maximum likelihood; maximum product of spacing; weighted least squares; stress-strength model

\section{Introduction}

A new extension of the Lomax distribution is proposed by considering the power transformation $X=$ $T^{\beta}$, where the random variable $T$ follows Lomax distribution with parameters $\alpha, \lambda$. The power Lomax (PL) distribution is obtained by Rady et al. (2016). A random variable $X$ is said to have PL distribution, if its probability density function (pdf) is given by

$$
\begin{aligned}
& f(x ; \alpha, \beta, \lambda)=\alpha \beta \lambda^{\alpha} x^{\beta-1}\left(\lambda+x^{\beta}\right)^{-\alpha-1} ; \alpha, \beta, \lambda>0, \\
& F(x ; \alpha, \beta, \lambda)=1-\lambda^{\alpha}\left(\lambda+x^{\beta}\right)^{-\alpha} ; \alpha, \beta, \lambda>0 .
\end{aligned}
$$

It was studied by many authors (Kumar et al., 2017; Mokhlis et al., 2017; Almetwaly and Almongy, 2018; Almetwally et al., 2018; El-Sherpieny et al., 2020). Figure 1 represents the different graphs of pdf of the PL distribution for different values of the parameters.

Ekström (2014) talked about maximum product of spacing (MPS) as an alternative to the maximum likelihood estimation (MLE) technique. Much of the time, the MPS technique works better than the MLE strategy and alluring properties, for example, consistency and asymptotic productivity of the MPS estimator firmly parallel those of the MLE when the last functions admirably. For more examples, see Singh et al. (2014); Almetwally and Almongy (2019b,a); AHmad and Almetwally (2020); El-Sherpieny et al. (2020).

The stress strength model has been known in the mechanical as follows, the stress is the mechanical loads and forces, while the strength is the physical effort that can resist the loads to perform its required function. Birnbaum (1956) was one of the first researchers who dealt with

\footnotetext{
${ }^{*}$ Corresponding author. Email: ehabxp_2009@hotmail.com.
} 

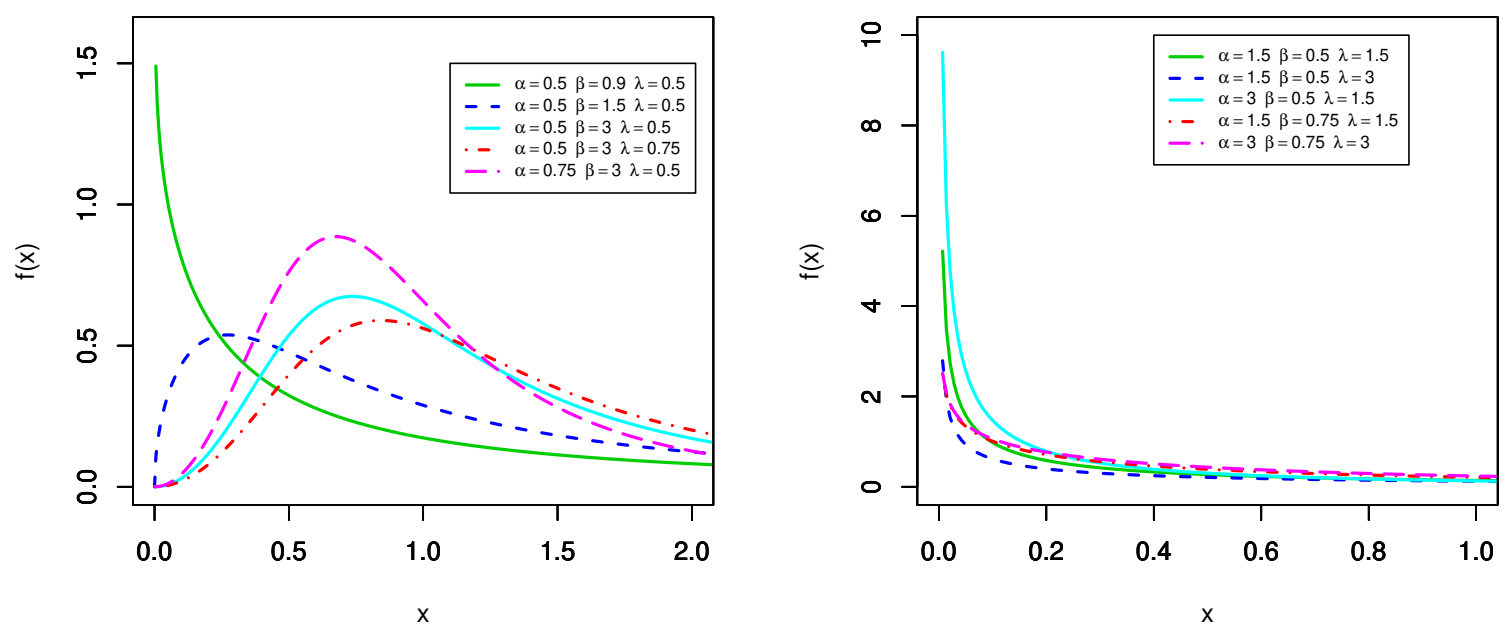

Figure 1: Different plots of the PL distribution for some parameter values.

the model of stress-strength content. When the stress exceeds the strength, the failure occurs. If $\mathrm{X}$ represents the strength and $\mathrm{Y}$ represents the stress, the main theme of statisticians is to estimate the probability of failure or reliability of this model, it is defined as $R=P(Y<X)$. Since the reliability concept is general, so the stress strength model can be applied in different fields outside of the scope of mechanics, for more details see Saraçoğlu et al. (2012). Singh et al. (2014) discussed the problem of the estimation of stressed system reliability under both classical and Bayesian paradigms. Mokhlis et al. (2017) presented characterizations, associated with the stress strength reliability of distributions with some general exponential and general inverse exponential forms.

There are two main objectives in this article: Firstly a comparison study of different estimation methods for the power Lomax distribution to conclude the best estimation method for parameters of PL distribution. Secondly propose the maximum product of spacing method as estimation method of reliability estimation for stress-strength model as an alternative method to maximum likelihood and Bayesian estimation methods, which we think would be of deep interest to statisticians. The rest of the paper is organized as follows. In Section 2, we propose the parameters estimation by using classical estimation and Bayesian estimation. In Section 3, we will use MLE, MPS and Bayesian estimation methods for parameter estimation of the stressstrength model. In Section 4, simulation study are given. Application of real data are discussed in Section 5. Finally, the conclusion and results are given in Section 6.

\section{Parameter Estimation}

In this section, we propose the parameters estimation by using classical estimation and Bayesian estimation. 


\subsection{Classical Estimation}

In this subsection, we propose the parameters estimation by utilizing traditional estimation. This methods are MLE, Ordinal Least Square (OLS), Weighted Least Squares (WLS), Cramér-von Mises (CVM) and MPS.

\subsubsection{Maximum Likelihood Estimation}

Rady et al. (2016) discussed the likelihood function of the PL distribution is

$$
L(\Phi)=\alpha^{n} \beta^{n} \lambda^{n \alpha} \prod_{i=1}^{n} x_{i}^{\beta-1}\left(\lambda+x_{i}^{\beta}\right)^{-\alpha-1},
$$

where $\Phi=(\alpha, \beta, \lambda)$, and the log likelihood function is given as

$$
l(\Phi)=n(\ln \alpha+\ln \beta+\alpha \ln \lambda)+(\beta-1) \sum_{i=1}^{n} \ln \left(x_{i}\right)-(\alpha+1) \sum_{i=1}^{n} \ln \left(\lambda+x_{i}^{\beta}\right) .
$$

The normal equations for the unknown parameters have been discussed by Rady et al. (2016). We can use any iterative procedure techniques such as Nelder-Mead type algorithms, to obtain as the numerical solution.

\subsubsection{Maximum Product Spacing}

As per Cheng and Amin (1983) presented MPS as following

$$
G=\left(\prod_{i=1}^{n+1} D_{i}\right)^{\frac{1}{n+1}}
$$

where $D_{i}$ can be written as follows

$$
D_{i}=\left\{\begin{array}{l}
D_{1}=F\left(x_{1}\right) \\
D_{i}=F\left(x_{i}-x_{i-1}\right) \quad i=2, \cdots, n \\
D_{n+1}=1-F\left(x_{n}\right)
\end{array}\right.
$$

such that $\sum D_{i}=1$, then the MPS estimators for PL distribution can be composed as pursues

$G(\Phi)=\left(\left(1-\lambda^{\alpha}\left(\lambda+x_{1}^{\beta}\right)^{-\alpha}\right)\left(\lambda^{\alpha}\left(\lambda+x_{n}^{\beta}\right)^{-\alpha}\right) \lambda^{\alpha} \prod_{i=2}^{n}\left[\left(\lambda+x_{i}^{\beta}\right)^{-\alpha}-\left(\lambda+x_{i-1}^{\beta}\right)^{-\alpha}\right]\right)^{\frac{1}{n+1}}$.

The natural logarithm of the product spacing function is

$$
\begin{aligned}
\ln G(\Phi)= & \frac{1}{n+1}\left[\ln \left(1-\lambda^{\alpha}\left(\lambda+x_{1}^{\beta}\right)^{-\alpha}\right)-\alpha \ln \left(\lambda+x_{n}^{\beta}\right)+2 \alpha \ln \lambda\right. \\
& \left.+\sum_{i=2}^{n} \ln \left(\left(\lambda+x_{i}^{\beta}\right)^{-\alpha}-\left(\lambda+x_{i-1}^{\beta}\right)^{-\alpha}\right)\right] .
\end{aligned}
$$


To obtain the normal equations for the unknown parameters, we differentiate Equation (8) partially with respect to the parameters $\Phi$ and equate them to zero. The estimators $\hat{\Phi}$ of $\Phi$ can be obtained as the solution of the following equations.

$$
\begin{aligned}
& \frac{\partial \ln G}{\partial \alpha}=\frac{1}{n+1}\left[\frac{\lambda^{\alpha}\left(\lambda+x_{1}^{\beta}\right)^{-\alpha}\left(\ln \left(\lambda+x_{1}^{\beta}\right)-\ln (\lambda)\right)}{\left(1-\lambda^{\alpha}\left(\lambda+x_{1}^{\beta}\right)^{-\alpha}\right)}-\ln \left(\lambda+x_{n}^{\beta}\right)+2 \ln (\lambda)\right. \\
& \left.+\sum_{i=2}^{n} \frac{\left(\left(\lambda+x_{i}^{\beta}\right)^{-\alpha} \ln \left(\lambda+x_{i}^{\beta}\right)\right)-\left(\left(\lambda+x_{i-1}^{\beta}\right)^{-\alpha} \ln \left(\lambda+x_{i-1}^{\beta}\right)\right)}{\left(\left(\lambda+x_{i}^{\beta}\right)^{-\alpha}-\left(\lambda+x_{i-1}^{\beta}\right)^{-\alpha}\right)}\right] \text {, } \\
& \frac{\partial \ln G}{\partial \lambda}=\frac{1}{n+1}\left[\frac{-\alpha \lambda^{\alpha-1}\left(\lambda+x_{1}^{\beta}\right)^{-\alpha-1} x_{1}^{\beta}}{\left(1-\lambda^{\alpha}\left(\lambda+x_{1}^{\beta}\right)^{-\alpha}\right)}\right. \\
& \left.+\alpha \sum_{i=2}^{n} \frac{\left(\left(\lambda+x_{i}^{\beta}\right)^{-\alpha-1}\right)-\left(\left(\lambda+x_{i-1}^{\beta}\right)^{-\alpha-1}\right)}{\left(\left(\lambda+x_{i}^{\beta}\right)^{-\alpha}-\left(\lambda+x_{i-1}^{\beta}\right)^{-\alpha}\right)}-\frac{\alpha}{\lambda+x_{n}^{\beta}}+\frac{2 \alpha}{\lambda}\right], \\
& \frac{\partial \ln G}{\partial \beta}=\frac{1}{n+1}\left[\frac{\alpha \lambda^{\alpha} x_{1}^{\beta}\left(\lambda+x_{1}^{\beta}\right)^{-\alpha-1} \ln \left(x_{1}\right)}{\left(1-\lambda^{\alpha}\left(\lambda+x_{1}^{\beta}\right)^{-\alpha}\right)}-\frac{\alpha x_{n}^{\beta} \ln \left(x_{n}\right)}{\left(\lambda+x_{n}^{\beta}\right)}\right. \\
& \left.+\alpha \sum_{i=2}^{n} \frac{\left(x_{i}^{\beta} \ln \left(x_{i}\right)\left(\lambda+x_{i}^{\beta}\right)^{-\alpha-1}\right)-\left(x_{i-1}^{\beta} \ln \left(x_{i-1}\right)\left(\lambda+x_{i-1}^{\beta}\right)^{-\alpha-1}\right)}{\left(\left(\lambda+x_{i}^{\beta}\right)^{-\alpha}-\left(\lambda+x_{i-1}^{\beta}\right)^{-\alpha}\right)}\right] .
\end{aligned}
$$

The above nonlinear equations cannot be solved analytically so, we can use any iterative procedure techniques such as Nelder-Mead type algorithms to obtain as the numerical solution.

\subsubsection{Weighted and Ordinary Least Square Method}

Swain et al. (1988) presented the OLS method for parameter estimation of distribution, it dependent on the observed sample $x_{1}<\cdots<x_{n}$ from be $n$ ordered random sample of any distribution with CDF, where $F($.$) denotes the \mathrm{CDF}$, we get

$$
E\left[F\left(x_{i}\right)\right]=\frac{i}{n+1} .
$$

The WLS method can be written as follows,

$$
P(\Phi)=\sum_{i=1}^{n} w_{i}\left(F\left(x_{i} ; \Phi\right)-\frac{i}{(n+1)}\right)^{2} .
$$


If $w_{i}=1$, then the estimate is OLS; if $w_{i}=\frac{(n+1)^{2}(n+2)}{i(n-i+1)}$, then the estimate is WLS. The WLS method of the PL distribution can be written as follows

$$
\operatorname{WLS}(\Phi)=\sum_{i=1}^{n} w_{i}\left(1-\lambda^{\alpha}\left(\lambda+x_{i}^{\beta}\right)^{-\alpha}-\frac{i}{(n+1)}\right)^{2} .
$$

After differentiating Equation (11) with respect to parameters $\Phi$ and then equating them to zero, we will get the following:

$$
\begin{aligned}
& \frac{\partial P_{\mathrm{WLS}}}{\partial \alpha}=2 \sum_{i=1}^{n} w_{i}\left[\left(1-\lambda^{\alpha}\left(\lambda+x_{i}^{\beta}\right)^{-\alpha}-\frac{i}{(n+1)}\right) \frac{\lambda^{\alpha}\left(\ln \left(\lambda+x_{i}^{\beta}\right)-\ln (\lambda)\right)}{\left(\lambda+x_{i}^{\beta}\right)^{\alpha}}\right], \\
& \frac{\partial P_{\mathrm{WLS}}}{\partial \beta}=2 \sum_{i=1}^{n} w_{i}\left[\left(1-\lambda^{\alpha}\left(\lambda+x_{i}^{\beta}\right)^{-\alpha}-\frac{i}{(n+1)}\right) \frac{\alpha \lambda^{\alpha} x_{i}^{\beta} \ln \left(x_{i}\right)}{\left(\lambda+x_{i}^{\beta}\right)^{\alpha+1}}\right], \\
& \frac{\partial P_{\mathrm{WLS}}}{\partial \lambda}=2 \sum_{i=1}^{n} w_{i}\left[\left(1-\lambda^{\alpha}\left(\lambda+x_{i}^{\beta}\right)^{-\alpha}-\frac{i}{(n+1)}\right) \frac{-\alpha \lambda^{\alpha-1} x_{i}^{\beta}}{\left(\lambda+x_{i}^{\beta}\right)^{\alpha+1}}\right] .
\end{aligned}
$$

The above nonlinear equations cannot be solved analytically so, we can use any iterative procedure techniques such as Nelder-Mead type algorithms to obtain as the numerical solution.

\subsubsection{Method of Cramér-von Mises}

The Cramér-von Mises estimates $\hat{\Phi}$ are obtained by minimizing with respect to $\Phi$ the function:

$$
\operatorname{CvM}(\Phi)=\frac{1}{12 n}+\sum_{i=1}^{n}\left(F\left(x_{i} ; \Phi\right)-\frac{2 i-1}{2 n}\right)^{2} .
$$

The estimators can also be obtained by solving the following nonlinear equations:

$$
\begin{aligned}
& \frac{\partial \operatorname{CvM}(\Phi)}{\partial \alpha}=2 \sum_{i=1}^{n}\left[\left(1-\lambda^{\alpha}\left(\lambda+x_{i}^{\beta}\right)^{-\alpha}-\frac{2 i-1}{2 n}\right) \frac{\lambda^{\alpha}\left(\ln \left(\lambda+x_{i}^{\beta}\right)-\ln (\lambda)\right)}{\left(\lambda+x_{i}^{\beta}\right)^{\alpha}}\right], \\
& \frac{\partial \operatorname{CvM}(\Phi)}{\partial \beta}=2 \sum_{i=1}^{n}\left[\left(1-\lambda^{\alpha}\left(\lambda+x_{i}^{\beta}\right)^{-\alpha}-\frac{2 i-1}{2 n}\right) \frac{\alpha \lambda^{\alpha} x_{i}^{\beta} \ln \left(x_{i}\right)}{\left(\lambda+x_{i}^{\beta}\right)^{\alpha}}\right], \\
& \frac{\partial \operatorname{CvM}(\Phi)}{\partial \lambda}=2 \sum_{i=1}^{n}\left[\left(1-\lambda^{\alpha}\left(\lambda+x_{i}^{\beta}\right)^{-\alpha}-\frac{2 i-1}{2 n}\right) \frac{-\alpha \lambda^{\alpha-1} x_{i}^{\beta}}{\left(\lambda+x_{i}^{\beta}\right)^{\alpha+1}}\right] .
\end{aligned}
$$

The above nonlinear equations cannot be solved analytically so, we can use any iterative procedure techniques such as Nelder-Mead type algorithms to obtain as the numerical solution. 


\subsection{Bayesian Estimation}

In this sectio, the Bayes estimate using square error and Linex loss functions of the unknown parameters $\Phi$ of the PL distribution will be obtained. The Bayes estimates is considered under the assumption that the random variables $\Phi$ have an independent gamma distribution. Assumed that $\Phi_{j} \sim \operatorname{Gamma}\left(a_{j}, b_{j}\right)$ then, the joint prior density of $\Phi$ can be written as

$$
g(\Phi) \propto \prod_{j=1}^{3} \Phi_{j}^{a_{j}} e^{-\frac{\Phi_{j}}{b_{j}}} ; a_{j}, b_{j}>0 ; j=1,2,3,
$$

here all the hyper parameters $a_{j}$ and $b_{j}$ are known and non-negative. For the choice of hyperparameters, the experimenters can incorporate their prior guess in terms of location and precision for the parameter of interest. Such that

$$
E\left(\Phi_{j}\right)=b_{j} a_{j} ; \operatorname{Var}\left(\Phi_{j}\right)=a_{j} b_{j}^{2} b^{2} .
$$

Combining Equation (3) and Equation (13) to obtain the posterior density of $\Phi$ take the following form

$$
g^{*}(\Phi \mid x)=\frac{L(\Phi) \cdot g(\Phi)}{\int_{\Phi} L(\Phi) \cdot g(\Phi) d \Phi} .
$$

Therefore, the Bayes estimates of the unknown parameters $\Phi$ under square error denoted by $\tilde{\Phi}$; can be calculated through the following equations as follows

$$
\tilde{\Phi}=E(\Phi \mid \underline{x})=\int_{0}^{\infty} \int_{0}^{\infty} \int_{0}^{\infty} \Phi g^{*}(\Phi \mid x) d \Phi_{1} d \Phi_{2} d \Phi_{3} .
$$

The Linex loss function has been introduced by Varian (1975). One of the most commonlyused asymmetric loss functions is the Linex loss function

$$
L\left(\hat{\Phi}_{j}\right)=e^{\mathcal{V}(\hat{\Phi}-\Phi)}-\mathcal{V}(\hat{\Phi}-\Phi)-1, \mathcal{V} \neq 0 .
$$

For $\mathcal{V}>0$, the overestimation is more serious than underestimation, for $\mathcal{V}<0$, the underestimation is more serious than the overestimation, and for $\mathcal{V}$ closed to zero, the Linex loss is approximately squared error loss and therefore almost symmetric. Almetwaly and Almongy (2018) used the Bayes estimator of $\Phi_{j}$, denoted by $\hat{\Phi}_{j}$ under Linex loss function has been introduced by Zellner (1986). The Linex loss function is given as following

$$
\tilde{\tilde{\Phi}}_{j}=\frac{-1}{\mathcal{V}} \ln \left(\sum_{i=1}^{M} \frac{e^{-\mathcal{V} \Phi_{j}^{(i)}}}{M}\right)
$$

For this situation, we utilize the MCMC strategy to generate samples from the posterior distributions and after that register the Bayes estimators of the individual parameters. In the Markov chain Monte Carlo (MCMC) technique can be used to generate samples from the posterior density function. The joint posterior density functions of $\Phi_{j} ; j=1,2,3$ can be written as

$$
g^{*}(\Phi \mid x) \propto \alpha^{n\left(a_{1}-1\right)} \beta^{n\left(a_{2}-1\right)} \lambda^{n \alpha\left(a_{3}-1\right)} \prod_{i=1}^{n} x_{i}^{\beta-1}\left(\lambda+x_{i}^{\beta}\right)^{-\alpha-1} \prod_{j=1}^{3} e^{-\frac{\Phi_{j}}{b_{j}}}
$$


For the PL distribution distributions, the full conditional posterior distributions of the parameters are given by

$$
\begin{aligned}
& g_{1}(\alpha \mid \beta, \lambda, x) \propto \alpha^{n\left(a_{1}-1\right)} e^{-\frac{\alpha}{b_{1}}} \prod_{i=1}^{n}\left(\lambda+x_{i}^{\beta}\right)^{-\alpha-1}, \\
& g_{2}(\beta \mid \alpha, \lambda, x) \propto \beta^{n\left(a_{2}-1\right)} e^{-\frac{\beta}{b_{2}}} \prod_{i=1}^{n} x_{i}^{\beta-1}\left(\lambda+x_{i}^{\beta}\right)^{-\alpha-1}, \\
& g_{3}(\lambda \mid \alpha, \beta, x) \propto \lambda^{n \alpha\left(a_{3}-1\right)} e^{-\frac{\lambda}{b_{3}}} \prod_{i=1}^{n}\left(\lambda+x_{i}^{\beta}\right)^{-\alpha-1} .
\end{aligned}
$$

Since the full conditional posterior distributions do not have simple forms in perspective of sampling, we use the Metropolis-Hastings algorithm within each Gibbs chain by Metropolis et al. (1953) and see example Almetwally et al. (2018) discussed Bayesian estimation for square error and Linex loss function have been used based on MCMC to estimate parameter of the Weibull Generalized Exponential Distribution under progressive censoring schemes.

In our simulation study presented in the next section, MCMC procedure is used to generate the full conditional posterior distributions. We set the number of periods in the MCMC process to be $M=10,000$.

\section{Stress-Strength Model}

For the stress-strength parameter of PL distribution. Let $X$ and $Y$ are the independent strength and stress random variables observed from PL. Then, the stress-strength reliability $R$ is calculated as:

$$
R=P(Y<X)=\int_{x=0}^{\infty}\left\{\int_{y=0}^{x} f\left(y ; \alpha_{2}, \lambda_{2}\right) d y\right\} f\left(x ; \alpha_{1}, \lambda_{1}\right) d x
$$

Then, we have

$$
\hat{R}=1-\alpha_{1} \beta_{1} \lambda_{1}^{\alpha_{1}} \lambda_{2}^{\alpha_{2}} \int_{0}^{\infty} x^{\beta_{1}-1}\left(\lambda_{1}+x^{\beta_{1}}\right)^{-\alpha_{1}-1}\left(\lambda_{2}+x^{\beta_{2}}\right)^{-\alpha_{2}} d x
$$

Rady et al. (2016) introduced the stress strength parameter $R$ given as follows

$$
\begin{aligned}
& \hat{R}=\alpha_{1} \beta_{1} \lambda_{1}^{\alpha_{1}}\left(1-\lambda_{2}^{-\beta_{2}\left(\alpha_{2}+1\right)} \lambda_{1}^{\beta_{1} \alpha_{1}}\right) \sum_{j=0}^{\infty}\left[\left(\begin{array}{c}
-\left(\alpha_{2}+1\right) \\
j
\end{array}\right)\right. \\
& \left(\lambda_{2}^{-j \beta_{2}} \frac{H G\left(\alpha_{1}, \frac{(1+j) \beta_{2}}{\beta_{1}}, 1+\frac{(1+j) \beta_{2}}{\beta_{1}},-\left(\frac{1}{\lambda_{1}}\right)^{\beta_{1}}\right)}{(1+j) \beta_{2}}+\right. \\
& \lambda_{2}^{\beta_{2}} \frac{H G\left(\alpha_{1}, \frac{\alpha_{1} \beta_{1}+\left(\alpha_{2}+j\right) \beta_{2}-1}{\beta_{1}}, 1+\frac{\left(\alpha_{1}+1\right) \beta_{1}+\left(\alpha_{2}+j\right) \beta_{2}-1}{\beta_{1}},-\left(\frac{1}{\lambda_{1}}\right)^{-\beta_{1}}\right)}{\alpha_{1} \beta_{1}+\left(\alpha_{2}+j\right) \beta_{2}-1} \\
& \left.\left.\left(\left(\frac{1}{\lambda_{1}}\right)^{-\beta_{1}}\right)^{\frac{\alpha_{1} \beta_{1}+\left(\alpha_{2}+j\right) \beta_{2}-1}{\beta_{1}}}\left(\left(\frac{1}{\lambda_{1}}\right)^{\beta_{1}}\right)^{\frac{\left(\alpha_{2}+j\right) \beta_{2}-1}{\beta_{1}}}\right)\right] \text {. }
\end{aligned}
$$



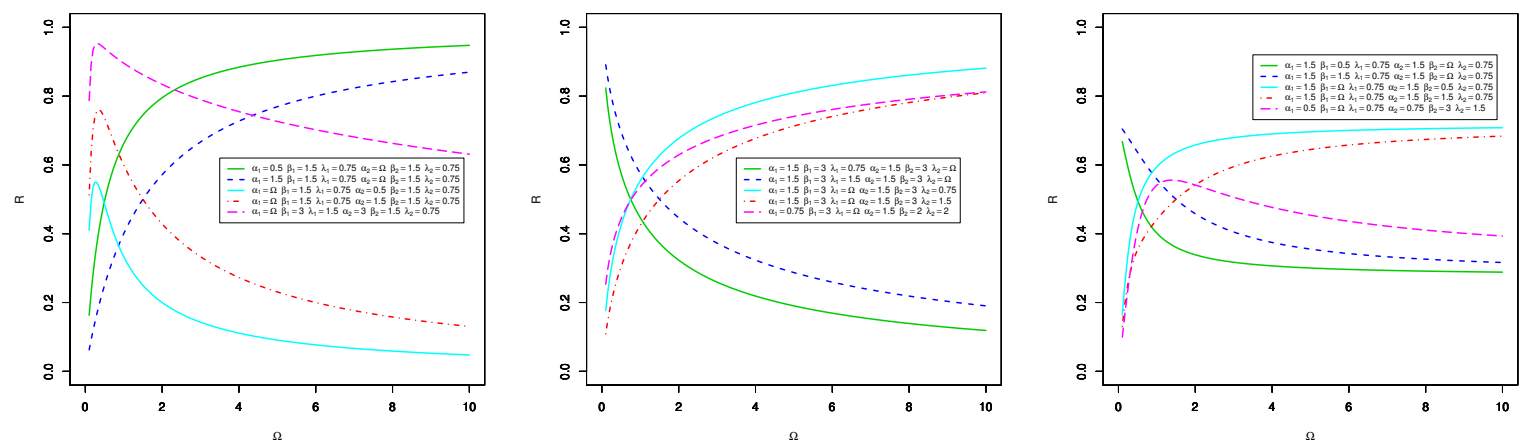

Figure 2: Plots of the PL reliability functions of stress-strength by some values of parameters.

Figure 2 display plots of the reliability functions of stress-strength for the PL distribution for different values of parameters, where the higher the values of the distribution parameters of the stress variable, the lower the reliability value. The higher the values of the parameters of the variable strength, the higher the reliability value, as shown in Figure 2.

We propose the method of MPS for reliability estimation of stress-strength model as alternative method to MLE and Bayesian estimation methods.

We will use possible classical estimation methods and Bayesian estimation methods for parameter estimation of the stress-strength model in case of complete sample of observations.

\subsection{Maximum Likelihood Estimation}

The likelihood function of PL distribution for stress-strength model is

$$
L(\mathcal{B})=\prod_{i=1}^{n} f\left(x_{i} ; \Phi_{1}\right) \prod_{j=1}^{m} f\left(y_{j} ; \Phi_{2}\right)
$$

and the log-likelihood function is given as

$$
\begin{aligned}
l(\mathcal{B})= & \ln L(\mathcal{B}) \\
= & n\left(\ln \alpha_{1}+\ln \beta_{1}+\alpha_{1} \ln \lambda_{1}\right)+\left(\beta_{1}-1\right) \sum_{i=1}^{n} \ln \left(x_{i}\right)-\left(\alpha_{1}+1\right) \sum_{i=1}^{n} \ln \left(\lambda_{1}+x_{i}^{\beta_{1}}\right) \\
& +m\left(\ln \alpha_{2}+\ln \beta_{2}+\alpha_{2} \ln \lambda_{2}\right)+\left(\beta_{2}-1\right) \sum_{j=1}^{m} \ln \left(y_{j}\right)-\left(\alpha_{2}+1\right) \sum_{j=1}^{m} \ln \left(\lambda_{2}+y_{j}^{\beta_{2}}\right) .
\end{aligned}
$$

To obtain the normal equations for the unknown parameters, we differentiate Equation (16) partially with respect to the parameters $\mathcal{B}$ where $\mathcal{B}=\left(\Phi_{1}, \Phi_{2}\right)=\left(\alpha_{1}, \beta_{1}, \lambda_{1}, \alpha_{2}, \beta_{2}, \lambda_{2}\right)$ and equate them to zero. The estimators $\hat{\mathcal{B}}$ can be obtained as the simultaneous solutions of the equations

$$
\frac{\partial l(B)}{\partial \alpha_{j}}=\frac{s_{j}}{\alpha_{j}}+s_{j} \ln \lambda_{j}-\sum_{i=1}^{s_{j}} \ln \left(\lambda_{j}+z_{j i}^{\beta_{j}}\right) ; j=1,2,
$$




$$
\begin{aligned}
& \frac{\partial l(B)}{\partial \beta_{j}}=\frac{s_{j}}{\beta_{j}}+\sum_{i=1}^{s_{j}} \ln \left(z_{j i}\right)-\left(\alpha_{j}+1\right) \sum_{i=1}^{s_{j}} \frac{z_{j i}^{\beta_{j}} \ln \left(z_{j i}\right)}{\left(\lambda_{j}+z_{j i}^{\beta_{j}}\right)} \\
& \frac{\partial l(B)}{\partial \lambda_{j}}=\frac{s_{j} \alpha_{j}}{\lambda_{j}}-\left(\alpha_{j}+1\right) \sum_{i=1}^{s_{j}} \frac{1}{\left(\lambda_{j}+z_{j i}^{\beta_{j}}\right)} .
\end{aligned}
$$

where $z_{j i}=\left(x_{i} y_{i}\right), s_{j}=(n m)$.

\subsection{Maximum Product Spacing}

The Maximum Product Spacing for stress-strength model is denoted as following

$$
\operatorname{GS}(\mathcal{B})=\left(\prod_{i=1}^{n+1} D_{i}\left(x_{i} ; \alpha_{1}, \lambda_{1}\right)\right)^{\frac{1}{n+1}}\left(\prod_{i=1}^{m+1} D_{i}\left(y_{j} ; \alpha_{2}, \lambda_{2}\right)\right)^{\frac{1}{m+1}}
$$

such that $\sum_{i} D_{i}\left(z_{j i} ; \alpha_{j}, \lambda_{j}\right)=1$, where

$$
D_{i}(B)=\left\{\begin{array}{l}
D_{1}=F\left(z_{j 1} ; \alpha_{j}, \lambda_{j}\right) \\
D_{i}=F\left(z_{j i} ; \alpha_{j}, \lambda_{j}\right)-F\left(z_{j(i-1)} ; \alpha_{j}, \lambda_{j}\right) i=2, \cdots, s_{j}, j=1,2 \\
\left.D_{n+1}=1-F\left(z_{j n} ; \alpha_{j}, \lambda_{j}\right)\right) .
\end{array}\right.
$$

The natural logarithm of the product spacing function of the exponential distribution for stress-strength model is denoted as following

$$
\begin{aligned}
\operatorname{gs}(\mathcal{B})= & \ln \operatorname{GS}(\mathcal{B}) \\
= & \frac{1}{n+1}\left[\ln \left(1-\lambda_{1}^{\alpha_{1}}\left(\lambda_{1}+x_{1}^{\beta_{1}}\right)^{-\alpha_{1}}\right)-\alpha_{1} \ln \left(\lambda_{1}+x_{n}^{\beta_{1}}\right)+2 \alpha_{1} \ln \lambda_{1}\right. \\
& \left.+\sum_{i=2}^{n} \ln \left(\left(\lambda_{1}+x_{i}^{\beta_{1}}\right)^{-\alpha_{1}}-\left(\lambda_{1}+x_{i-1}^{\beta_{1}}\right)^{-\alpha_{1}}\right)\right] \\
& +\frac{1}{m+1} \ln \left(1-\lambda_{2}^{\alpha_{2}}\left(\lambda_{2}+y_{1}^{\beta_{2}}\right)^{-\alpha_{2}}\right)-\alpha_{2} \ln \left(\lambda_{2}+y_{m}^{\beta_{2}}\right)+2 \alpha_{2} \ln \lambda_{2} \\
& +\sum_{i=2}^{m} \ln \left(\left(\lambda_{2}+y_{i}^{\beta_{2}}\right)^{-\alpha_{2}}-\left(\lambda_{2}+y_{i-1}^{\beta_{2}}\right)^{-\alpha_{2}}\right) .
\end{aligned}
$$

To obtain the normal equations for the unknown parameters, we differentiate Equation (20)

partially with respect to the parameters $\mathcal{B}$ and equate them to zero. The estimators $\hat{\mathcal{B}}$ can be obtained as the solution of the following equations.

$$
\begin{aligned}
\frac{\partial \operatorname{gs}(\mathcal{B})}{\partial \alpha_{j}}= & \frac{1}{s_{j}+1}\left[\frac{\lambda_{j}^{\alpha_{j}}\left(\lambda_{j}+z_{j 1}^{\beta_{j}}\right)^{-\alpha_{j}}\left(\ln \left(\lambda_{j}+z_{j 1}^{\beta_{j}}\right)-\ln \left(\lambda_{j}\right)\right)}{\left(1-\lambda_{j}^{\alpha_{j}}\left(\lambda_{j}+z_{j 1}^{\beta_{j}}\right)^{-\alpha_{j}}\right)}-\ln \left(\lambda_{j}+z_{j n}^{\beta_{j}}\right)+2 \ln \left(\lambda_{j}\right)\right. \\
& \left.+\sum_{i=2}^{n} \frac{\left(\left(\lambda_{j}+z_{j i}^{\beta_{j}}\right)^{-\alpha_{j}} \ln \left(\lambda_{j}+z_{j i}^{\beta_{j}}\right)\right)-\left(\left(\lambda_{j}+z_{j i-1}^{\beta_{j}}\right)^{-\alpha_{j}} \ln \left(\lambda_{j}+z_{j i-1}^{\beta_{j}}\right)\right)}{\left(\left(\lambda_{j}+z_{j i}^{\beta_{j}}\right)^{-\alpha_{j}}-\left(\lambda_{j}+z_{j i-1}^{\beta_{j}}\right)^{-\alpha_{j}}\right)}\right],
\end{aligned}
$$




$$
\begin{aligned}
\frac{\partial \operatorname{gs}(\mathcal{B})}{\partial \beta_{j}}= & \frac{1}{s_{j}+1}\left[\frac{\alpha_{j} \lambda_{j}^{\alpha_{j}} z_{j 1}^{\beta_{j}}\left(\lambda_{j}+z_{j 1}^{\beta_{j}}\right)^{-\alpha_{j}-1} \ln \left(z_{j 1}\right)}{\left(1-\lambda_{j}^{\alpha_{j}}\left(\lambda_{j}+z_{j 1}^{\beta_{j}}\right)^{-\alpha_{j}}\right)}-\frac{\alpha_{j} z_{j n}^{\beta_{j}} \ln \left(z_{j n}\right)}{\left(\lambda_{j}+z_{j n}^{\beta_{j}}\right)}\right. \\
& \left.+\alpha_{j} \sum_{i=2}^{n} \frac{\left(z_{j i}^{\beta_{j}} \ln \left(z_{j i}\right)\left(\lambda_{j}+z_{j i}^{\beta_{j}}\right)^{-\alpha_{j}-1}\right)-\left(z_{j i-1}^{\beta_{j}} \ln \left(z_{j i-1}\right)\left(\lambda_{j}+z_{j i-1}^{\beta_{j}}\right)^{-\alpha_{j}-1}\right)}{\left.\beta_{j}-\left(\lambda_{j}+z_{j i-1}^{\beta_{j}}\right)^{-\alpha_{j}}\right)}\right], \\
\frac{\partial \operatorname{gs}(\mathcal{B})}{\partial \lambda_{j}}= & \frac{1}{s_{j}+1}\left[\frac{-\alpha_{j} \lambda_{j}^{\alpha_{j}-1}\left(\lambda_{j}+z_{j 1}^{\beta_{j}}\right)^{-\alpha_{j}-1} z_{j 1}^{\beta_{j}}}{\left(1-\lambda_{j}^{\alpha_{j}}\left(\lambda_{j}+z_{j 1}^{\beta_{j}}\right)^{-\alpha_{j}}\right)}\right] \\
& \left.+\alpha_{j} \sum_{i=2}^{n} \frac{\left(\lambda_{j}+z_{j i}^{\beta_{j}}\right)^{-\alpha_{j}-1}-\left(\lambda_{j}+z_{j i-1}^{\beta_{j}}\right)^{-\alpha_{j}-1}}{\left(\left(\lambda_{j}+z_{j i}^{\beta_{j}}\right)^{-\alpha_{j}}-\left(\lambda_{j}+z_{j i-1}^{\beta_{j}}\right)^{-\alpha_{j}}\right)}-\frac{\alpha_{j}}{\lambda_{j}+z_{j n}^{\beta_{j}}}+\frac{2 \alpha_{j}}{\lambda_{j}}\right] .
\end{aligned}
$$

\subsection{Bayesian Estimation}

In this subsection, we discuss the Bayesian inference of the unknown parameters of a PL distribution under stress-strength model. For Bayesian parameters estimation we will consider squared error loss function. When the parameters of the model are unknown, a joint conjugate prior for the parameters does not exist. We suggest using independent gamma priors for $\mathcal{B}$ having pdfs. The joint prior density of $\mathcal{B}$ can be written as

$$
g(\mathcal{B}) \propto \prod_{j=1}^{2} \alpha_{j}^{a_{j}-1} e^{-\frac{\alpha_{j}}{b_{j}}} \beta_{j}^{c_{j}-1} e^{-\frac{\beta_{j}}{d_{j}}} \lambda_{j}^{f_{j}-1} e^{-\frac{\lambda_{j}}{g_{j}}} ; a_{j}, b_{j}, c_{j}, d_{j}, f_{j}, g_{j}>0, j=1,2 .
$$

By using MCMC technique generate samples from the posterior density function. The joint posterior density functions of $\mathcal{B}$ can be written as

$$
\begin{aligned}
g^{*}(B \mid x, y) \propto \alpha_{1}^{n\left(a_{1}-1\right)} \beta_{1}^{n\left(c_{1}-1\right)} \lambda_{1}^{n \alpha_{1}\left(f_{1}-1\right)} \prod_{i=1}^{n} x_{i}^{\beta_{1}-1}\left(\lambda_{1}+x_{i}^{\beta_{1}}\right)^{-\alpha_{1}-1} \\
\prod_{j=1}^{3} e^{-\frac{\Phi_{1}}{X_{1}}} \alpha_{2}^{m\left(a_{2}-1\right)} \beta_{2}^{m\left(c_{2}-1\right)} \lambda_{2}^{m \alpha_{2}\left(f_{2}-1\right)} \prod_{i=1}^{m} y_{i}^{\beta_{2}-1}\left(\lambda_{2}+y_{i}^{\beta_{2}}\right)^{-\alpha_{2}-1} \prod_{j=1}^{3} e^{-\frac{\Phi_{2}}{X_{2}}} .
\end{aligned}
$$

\section{Simulation Study}

In this portion, we give a complete algorithm of Monte-Carlo simulation study. We clarify our calculation through an application of PL distribution, particularly; we will utilize Monte-Carlo simulation study to discuss two aims.

Firstly, in the simulation of Section 2, the classical estimation methods and Bayesian estimation techniques are discussed to obtain the best estimation method of PL distribution. We can generate simulation by using $\mathrm{R}$ program. In this case, we must follow the following steps by order: 
Step 1: Suppose different values of the parameters vector of PL distribution.

Step 2: Choose the sample size $n=90,150$ and 300 .

Step 3: Generate the sample random values of PL distribution by using quantile function in equation $x_{i}=\left(\lambda\left(\left(1-u_{i}\right)^{\frac{-1}{\alpha}}-1\right)\right)^{\frac{1}{\beta}} ; 0<u_{i}<1$.

Step 4: Solve differential equations for each estimation methods, to obtain the estimators of the parameters for PL distribution, we calculate $\hat{\Phi}$.

Step 5: Repeat this experiment $(L-1)$ times. In each experiment, the same values of the parameters. It is certain that, the values of generating random are varying from experiment to experiment even though $\mathrm{n}$ are not changed.

In the end, we have L-values of Bias and MSE, we restricted the number of repeat this experiment to 10000. Take the averages of these values and call them Monte Carlo estimates: where $\hat{\Phi}$ is the estimated value of $\Phi$, Bias $=\operatorname{Mean}(\hat{\Phi}-\Phi)$, and the mean squared error (MSE) of the estimator. $\mathrm{MSE}=\operatorname{Mean}(\hat{\Phi}-\Phi)^{2}$.

Secondly, in stress-strength model, the MLE, MPS and Bayesian estimation has been obtained to get the best estimation method of parameters for PL distribution under stress-strength model. We can generate simulation by using $\mathrm{R}$ program. In this case, we must follow the following steps by order:

Step 1: Suppose different values of the parameters vector of PL distribution under stressstrength model as

Case 1: $\alpha_{1}=0.3, \beta_{1}=1.5, \lambda_{1}=0.75, \alpha_{2}=0.8, \beta_{2}=2, \lambda_{2}=0.8$ and

Case 2: $\alpha_{1}=0.25, \beta_{1}=1.5, \lambda_{1}=0.75, \alpha_{2}=1.5, \beta_{2}=1.5, \lambda_{2}=0.75$

Step 2: Choose the sample size of strength and stress variables as respectively

$n=50,80,100,140,150$ and 200 and $m=50,90,100,150$ and 230 .

Step 3: Generate the sample random values of PL distribution by using quantile function in equation $x_{1 i}=\left(\lambda_{1}\left(\left(1-u_{1 i}\right)^{\frac{-1}{\alpha_{1}}}-1\right)\right)^{\frac{1}{\beta_{1}}} ; 0<u_{1 i}<1$ and $x_{2 i}=\left(\lambda_{2}\left(\left(1-u_{2 i}\right)^{\frac{-1}{\alpha_{2}}}-1\right)\right)^{\frac{1}{\beta_{2}}} ; 0<u_{2 i}<1$.

Step 4: Solve differential equations in Section 3, to obtain the estimators of the parameters for PL distribution under stress-strength model, we calculate $\hat{B}$.

Step 5: Repeat this experiment (L) times. In each experiment, the same values of the parameters.

Step 6: According Rady et al. (2016), we calculated the stress-strength reliability by using Equation (15).

In the end, we have L-values of mean and MSE, we restricted the number of repeat this experiment to 10000 . Take the averages of these values and call them Monte Carlo estimates: where $\hat{B}$ is the estimated value of $\mathcal{B}$, and the mean squared error (MSE) of the estimator. $\operatorname{MSE}=\operatorname{Mean}(\hat{B}-\mathcal{B})^{2}$.

The following conclusions can be drawn from these Table 1-5 and Figure 3-4.

1. All the estimates reveal the property of consistency, i.e., the Bias and MSE decrease when $n$ increase.

2. Keeping $\beta$ and $\lambda$, the Bias and MSE of $\hat{\alpha}$ increases and the Bias and MSE of $\hat{\beta}$ decreases when $\alpha$ increases as shown in Table 1 .

3. Keeping $\alpha, \lambda$, the Bias and MSE of $\hat{\beta}$ increases and the Bias and MSE of $\hat{\alpha}$ and $\hat{\lambda}$ decreases when $\beta$ increases as shown in Table 2 .

4. In most cases, keeping $\alpha$, and $\beta$, the Bias and MSE of $\hat{\lambda}$ increases and the Bias and MSE of $\hat{\beta}$ decreases when $\lambda$ increases as shown in Table 3 . 

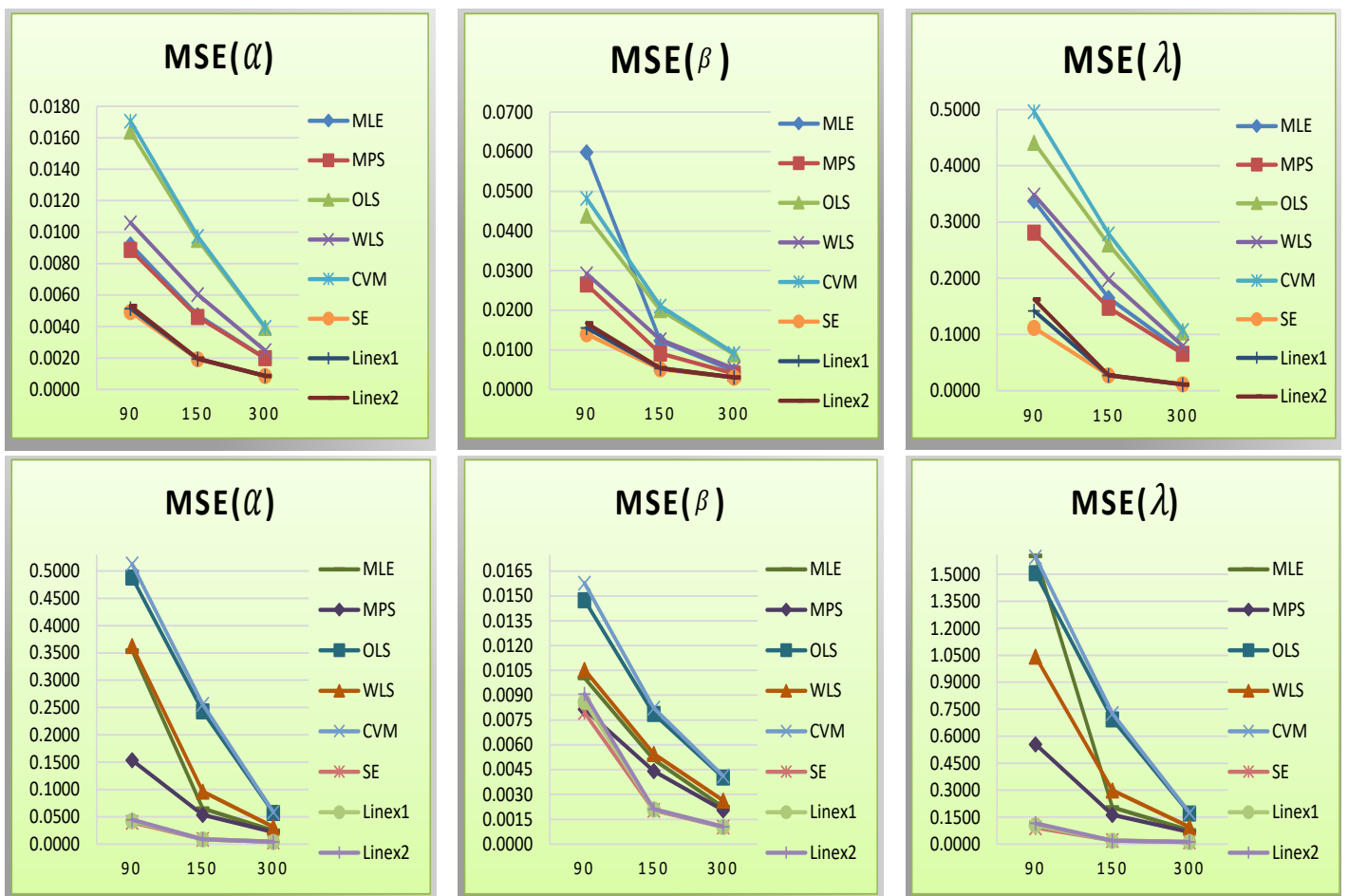

Figure 3: MSE for classical estimation methods and Bayesian estimation of PL parameters with different values of $\alpha$.
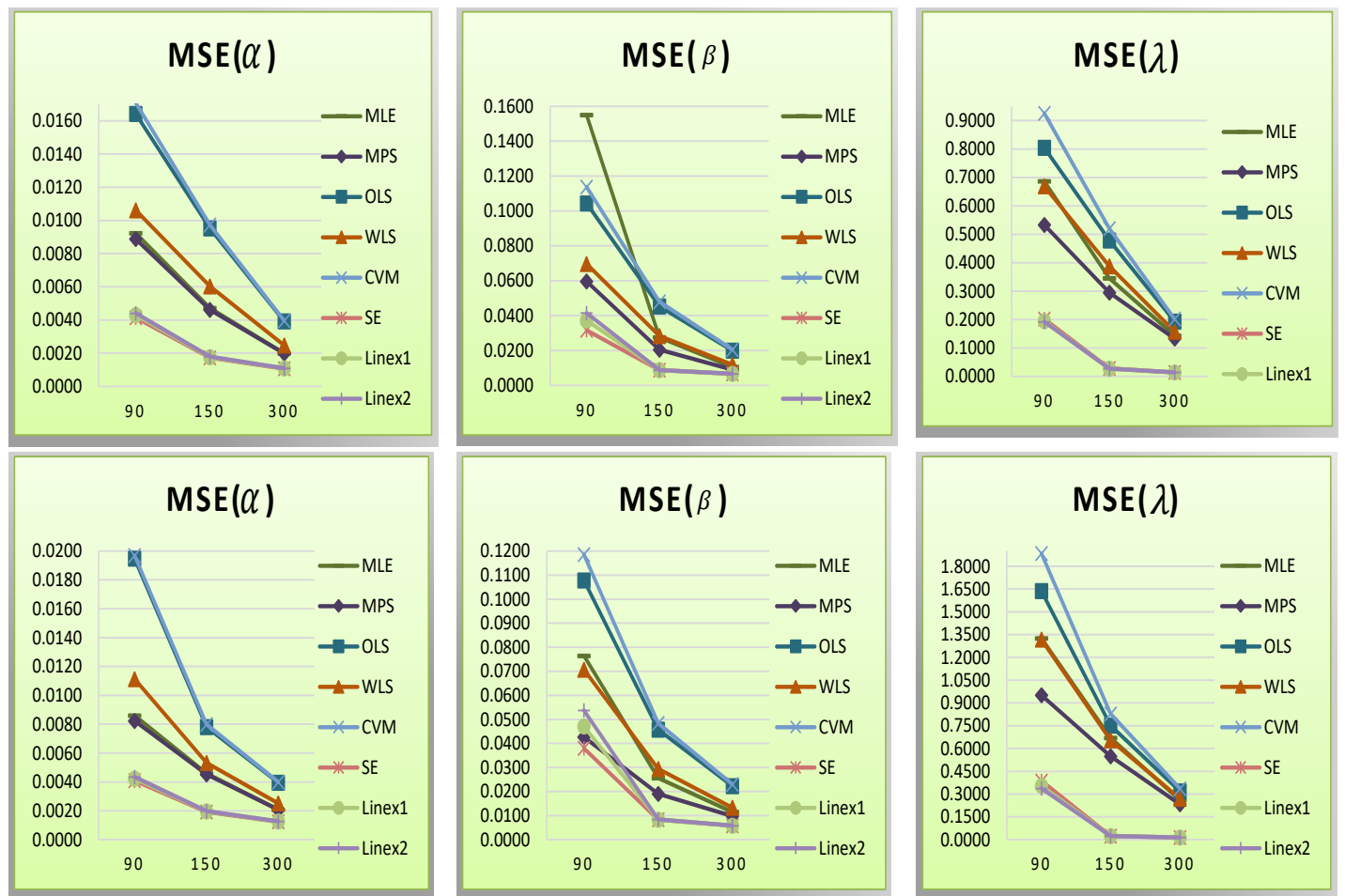

Figure 4: MSE for classical estimation methods and Bayesian estimation of PL parameters with different values of $\lambda$. 


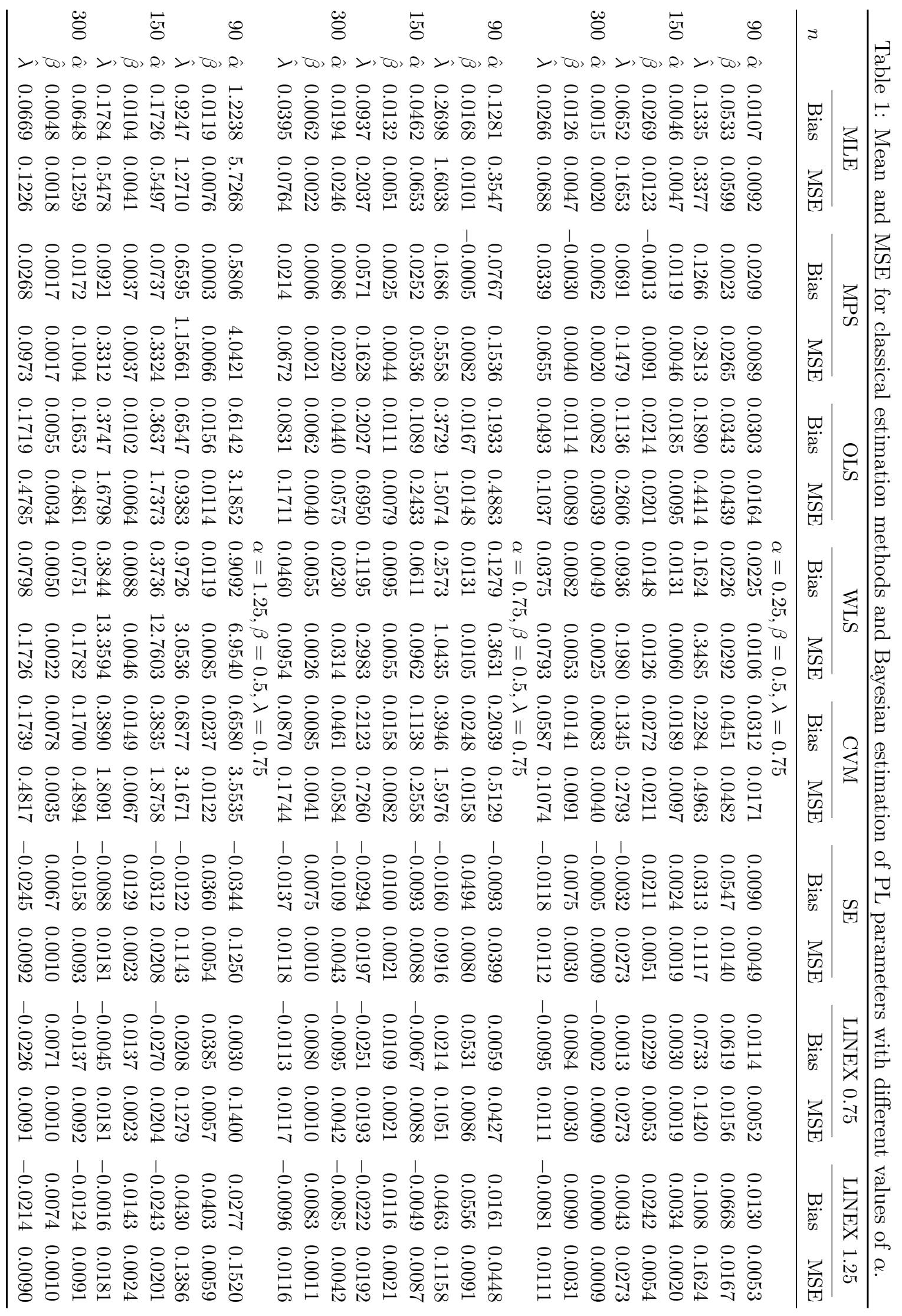




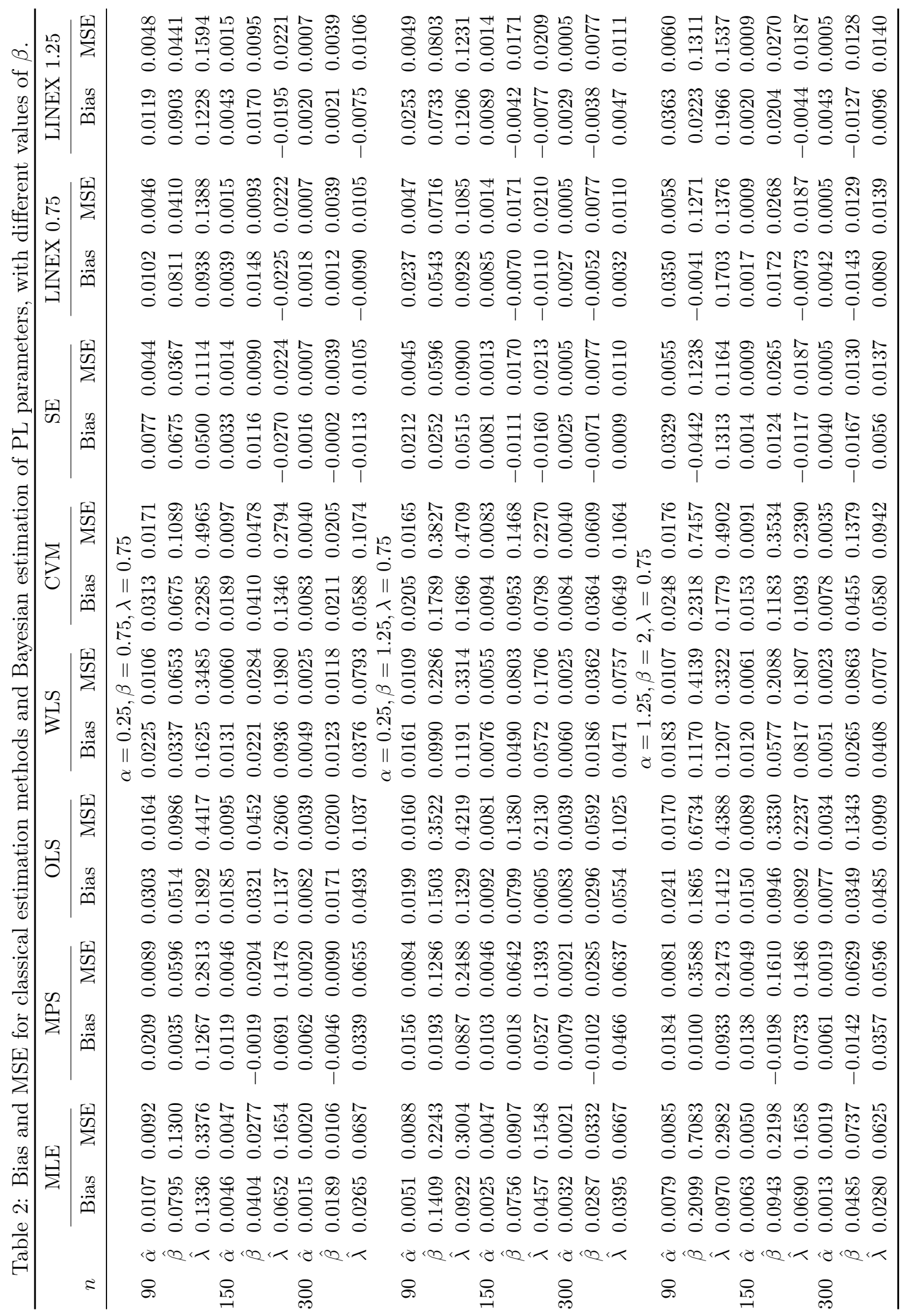




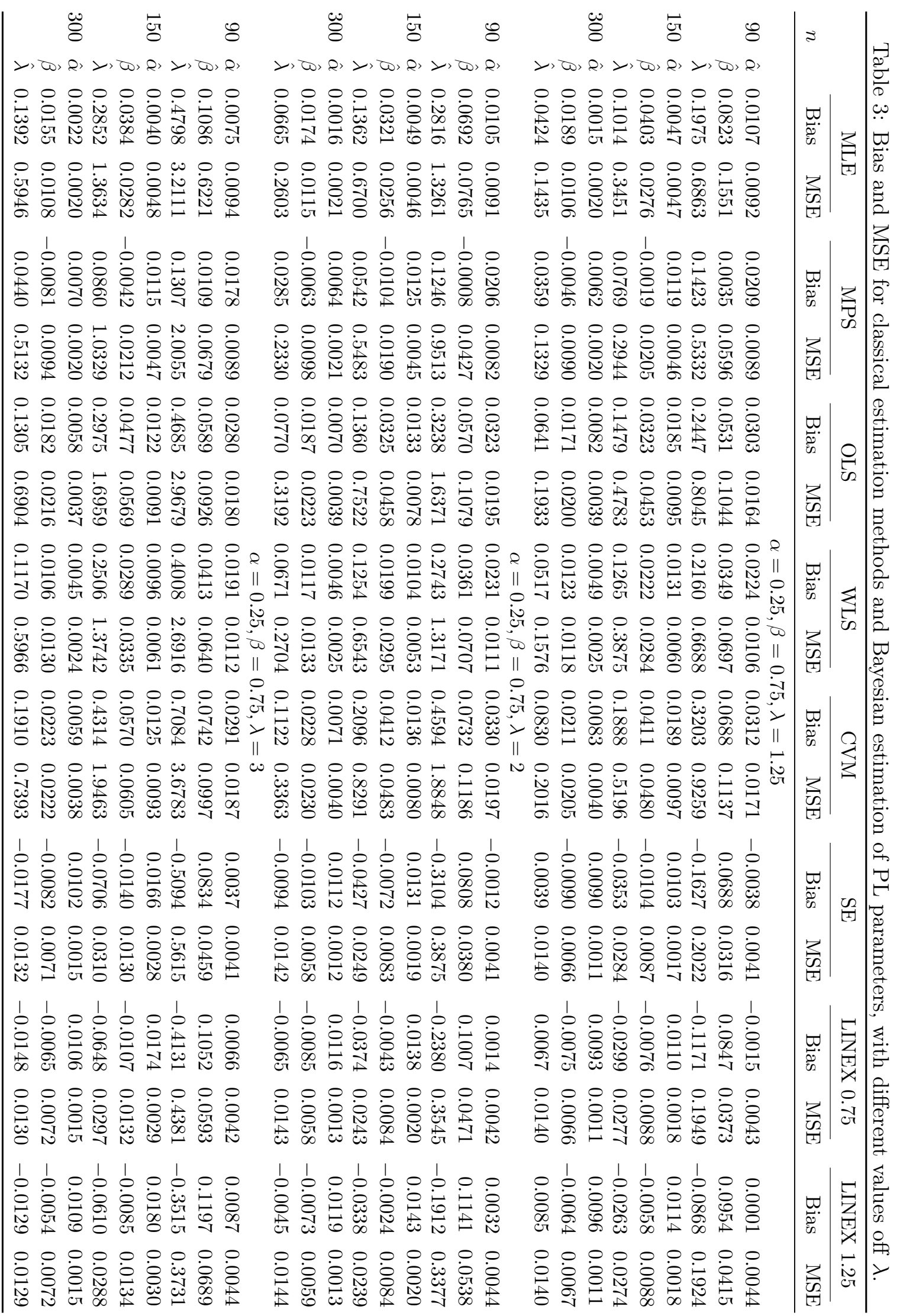


Table 4: Mean and MSE for MLE, MPS and Bayesian of PL parameters for stress-strength model.

\begin{tabular}{|c|c|c|c|c|c|c|c|c|c|}
\hline \multirow[b]{2}{*}{$(n, m)$} & \multirow[b]{2}{*}{ Methods } & & \multicolumn{7}{|c|}{$\alpha_{1}=0.3, \beta_{1}=1.5, \lambda_{1}=0.75, \alpha_{1}=0.8, \beta_{1}=2, \lambda_{1}=0.8$} \\
\hline & & & $\hat{\alpha}_{1}$ & $\hat{\beta}_{1}$ & $\hat{\lambda}_{1}$ & $\hat{\alpha}_{2}$ & $\hat{\beta}_{2}$ & $\hat{\lambda}_{2}$ & $\mathrm{R}$ \\
\hline \multirow[t]{6}{*}{$(50,50)$} & MLE & mean & 0.368 & 1.675 & 1.015 & 0.890 & 2.057 & 0.994 & 0.730 \\
\hline & & MSE & 0.417 & 0.496 & 2.436 & 0.136 & 0.126 & 0.461 & 0.002 \\
\hline & MPS & mean & 0.351 & 1.506 & 0.988 & 0.852 & 1.991 & 0.922 & 0.722 \\
\hline & & MSE & 0.059 & 0.260 & 1.132 & 0.100 & 0.098 & 0.320 & 0.002 \\
\hline & Bayes & mean & 0.308 & 1.471 & 0.717 & 0.794 & 1.988 & 0.791 & 0.718 \\
\hline & & MSE & 0.003 & 0.026 & 0.028 & 0.008 & 0.021 & 0.019 & 0.002 \\
\hline \multirow[t]{6}{*}{$(80,90)$} & MLE & mean & 0.317 & 1.619 & 0.870 & 0.852 & 2.011 & 0.906 & 0.728 \\
\hline & & MSE & 0.014 & 0.267 & 0.341 & 0.059 & 0.075 & 0.193 & 0.002 \\
\hline & MPS & mean & 0.323 & 1.501 & 0.847 & 0.829 & 1.975 & 0.866 & 0.723 \\
\hline & & MSE & 0.014 & 0.182 & 0.280 & 0.047 & 0.068 & 0.148 & 0.001 \\
\hline & Bayes & mean & 0.304 & 1.479 & 0.711 & 0.790 & 1.990 & 0.786 & 0.720 \\
\hline & & MSE & 0.002 & 0.023 & 0.023 & 0.007 & 0.016 & 0.019 & 0.001 \\
\hline \multirow[t]{6}{*}{$(100,100)$} & MLE & mean & 0.317 & 1.567 & 0.873 & 0.849 & 2.022 & 0.907 & 0.730 \\
\hline & & MSE & 0.010 & 0.129 & 0.243 & 0.057 & 0.057 & 0.185 & 0.001 \\
\hline & MPS & mean & 0.325 & 1.470 & 0.865 & 0.824 & 1.994 & 0.863 & 0.726 \\
\hline & & MSE & 0.011 & 0.093 & 0.212 & 0.042 & 0.050 & 0.136 & 0.001 \\
\hline & Bayes & mean & 0.304 & 1.489 & 0.732 & 0.790 & 1.999 & 0.791 & 0.724 \\
\hline & & MSE & 0.002 & 0.014 & 0.015 & 0.005 & 0.011 & 0.013 & 0.001 \\
\hline \multirow[t]{8}{*}{$(140,150)$} & MLE & mean & 0.310 & 1.557 & 0.844 & 0.834 & 2.009 & 0.868 & 0.733 \\
\hline & & MSE & 0.007 & 0.097 & 0.172 & 0.030 & 0.033 & 0.094 & 0.001 \\
\hline & MPS & mean & 0.316 & 1.487 & 0.839 & 0.823 & 1.985 & 0.849 & 0.730 \\
\hline & & MSE & 0.007 & 0.087 & 0.153 & 0.027 & 0.031 & 0.084 & 0.001 \\
\hline & Bayes & mean & 0.301 & 1.492 & 0.727 & 0.796 & 1.999 & 0.790 & 0.726 \\
\hline & & MSE & 0.001 & 0.013 & 0.015 & 0.004 & 0.009 & 0.010 & 0.001 \\
\hline & MLE & mean & 0.303 & 1.573 & 0.811 & 0.835 & 2.003 & 0.858 & 0.734 \\
\hline & & MSE & 0.005 & 0.085 & 0.137 & 0.034 & 0.035 & 0.096 & 0.001 \\
\hline \multirow[t]{6}{*}{$(150,150)$} & MPS & mean & 0.310 & 1.498 & 0.816 & 0.825 & 1.980 & 0.842 & 0.731 \\
\hline & & MSE & 0.006 & 0.063 & 0.132 & 0.033 & 0.034 & 0.092 & 0.001 \\
\hline & Bayes & mean & 0.301 & 1.491 & 0.742 & 0.800 & 1.993 & 0.794 & 0.729 \\
\hline & & MSE & 0.001 & 0.007 & 0.006 & 0.003 & 0.005 & 0.006 & 0.000 \\
\hline & MLE & mean & 0.302 & 1.563 & 0.778 & 0.807 & 2.027 & 0.813 & 0.731 \\
\hline & & MSE & 0.005 & 0.067 & 0.108 & 0.018 & 0.026 & 0.050 & 0.001 \\
\hline \multirow[t]{4}{*}{$(200,230)$} & MPS & mean & 0.307 & 1.507 & 0.782 & 0.799 & 2.011 & 0.801 & 0.729 \\
\hline & & MSE & 0.005 & 0.055 & 0.098 & 0.016 & 0.025 & 0.046 & 0.001 \\
\hline & Bayes & mean & 0.302 & 1.497 & 0.742 & 0.800 & 2.001 & 0.801 & 0.728 \\
\hline & & MSE & 0.001 & 0.006 & 0.007 & 0.002 & 0.004 & 0.006 & 0.000 \\
\hline
\end{tabular}


Table 5: Mean and MSE for MLE, MPS and Bayesian of PL parameters for stress-strength model.

\begin{tabular}{|c|c|c|c|c|c|c|c|c|c|}
\hline \multirow[b]{2}{*}{$(n, m)$} & \multirow[b]{2}{*}{ Methods } & & \multicolumn{7}{|c|}{$\alpha_{1}=0.25, \beta_{1}=1.5, \lambda_{1}=0.75, \alpha_{2}=1.5, \beta_{2}=1.5, \lambda_{2}=0.75$} \\
\hline & & & $\hat{\alpha}_{1}$ & $\hat{\beta}_{1}$ & $\hat{\lambda}_{1}$ & $\hat{\alpha}_{2}$ & $\hat{\beta}_{2}$ & $\hat{\lambda}_{2}$ & $\mathrm{R}$ \\
\hline \multirow[t]{6}{*}{$(50,50)$} & MLE & mean & 0.255 & 1.833 & 0.945 & 1.951 & 1.549 & 1.116 & 0.84 \\
\hline & & MSE & 0.017 & 0.835 & 0.842 & 3.249 & 0.057 & 2.015 & 0.00 \\
\hline & MPS & mean & 0.265 & 1.596 & 0.867 & 1.670 & 1.516 & 0.908 & 0.83 \\
\hline & & MSE & 0.014 & 0.487 & 0.453 & 1.175 & 0.049 & 0.748 & 0.00 \\
\hline & Bayes & mean & 0.253 & 1.470 & 0.704 & 1.471 & 1.513 & 0.728 & 0.83 \\
\hline & & MSE & 0.002 & 0.024 & 0.030 & 0.021 & 0.013 & 0.017 & 0.00 \\
\hline \multirow[t]{6}{*}{$(80,90)$} & MLE & mean & 0.260 & 1.634 & 0.863 & 1.741 & 1.524 & 0.950 & 0.84 \\
\hline & & MSE & 0.009 & 0.298 & 0.307 & 1.399 & 0.028 & 1.000 & 0.00 \\
\hline & MPS & mean & 0.272 & 1.486 & 0.857 & 1.593 & 1.508 & 0.845 & 0.83 \\
\hline & & MSE & 0.009 & 0.200 & 0.249 & 0.608 & 0.026 & 0.406 & 0.00 \\
\hline & Bayes & mean & 0.258 & 1.462 & 0.724 & 1.476 & 1.507 & 0.742 & 0.83 \\
\hline & & MSE & 0.001 & 0.021 & 0.021 & 0.018 & 0.007 & 0.013 & 0.00 \\
\hline \multirow[t]{6}{*}{$(100,100)$} & MLE & mean & 0.250 & 1.660 & 0.823 & 1.732 & 1.508 & 0.936 & 0.84 \\
\hline & & MSE & 0.007 & 0.259 & 0.249 & 0.649 & 0.022 & 0.428 & 0.00 \\
\hline & MPS & mean & 0.260 & 1.531 & 0.826 & 1.614 & 1.496 & 0.853 & 0.84 \\
\hline & & MSE & 0.007 & 0.165 & 0.217 & 0.456 & 0.021 & 0.297 & 0.00 \\
\hline & Bayes & mean & 0.252 & 1.483 & 0.724 & 1.481 & 1.510 & 0.734 & 0.84 \\
\hline & & MSE & 0.001 & 0.013 & 0.016 & 0.011 & 0.008 & 0.009 & 0.00 \\
\hline \multirow[t]{6}{*}{$(140,150)$} & MLE & mean & 0.259 & 1.560 & 0.826 & 1.595 & 1.508 & 0.834 & 0.84 \\
\hline & & MSE & 0.005 & 0.121 & 0.161 & 0.215 & 0.017 & 0.144 & 0.00 \\
\hline & MPS & mean & 0.266 & 1.475 & 0.824 & 1.526 & 1.496 & 0.787 & 0.84 \\
\hline & & MSE & 0.005 & 0.091 & 0.144 & 0.144 & 0.015 & 0.099 & 0.00 \\
\hline & Bayes & mean & 0.255 & 1.478 & 0.731 & 1.479 & 1.500 & 0.739 & 0.84 \\
\hline & & MSE & 0.001 & 0.013 & 0.015 & 0.011 & 0.005 & 0.008 & 0.00 \\
\hline \multirow[t]{6}{*}{$(150,150)$} & MLE & mean & 0.254 & 1.566 & 0.806 & 1.610 & 1.511 & 0.839 & 0.84 \\
\hline & & MSE & 0.005 & 0.097 & 0.152 & 0.221 & 0.016 & 0.144 & 0.00 \\
\hline & MPS & mean & 0.261 & 1.484 & 0.806 & 1.547 & 1.501 & 0.798 & 0.84 \\
\hline & & MSE & 0.004 & 0.073 & 0.130 & 0.173 & 0.015 & 0.112 & 0.00 \\
\hline & Bayes & mean & 0.252 & 1.490 & 0.736 & 1.490 & 1.500 & 0.742 & 0.84 \\
\hline & & MSE & 0.001 & 0.007 & 0.007 & 0.006 & 0.004 & 0.004 & 0.00 \\
\hline \multirow[t]{6}{*}{$(200,230)$} & MLE & mean & 0.250 & 1.563 & 0.776 & 1.585 & 1.505 & 0.820 & 0.84 \\
\hline & & MSE & 0.003 & 0.078 & 0.104 & 0.124 & 0.011 & 0.084 & 0.00 \\
\hline & MPS & mean & 0.256 & 1.495 & 0.784 & 1.545 & 1.498 & 0.795 & 0.84 \\
\hline & & MSE & 0.003 & 0.060 & 0.096 & 0.122 & 0.010 & 0.084 & 0.00 \\
\hline & Bayes & mean & 0.251 & 1.489 & 0.734 & 1.491 & 1.501 & 0.742 & 0.84 \\
\hline & & MSE & 0.001 & 0.006 & 0.007 & 0.005 & 0.003 & 0.004 & 0.00 \\
\hline
\end{tabular}


5. The Bayesian estimation method under SE loss function is best estimation method for parameters of PL distribution, Followed by Linex when $v=0.75$, next LINEX when $v=1.25$ and MPS method is superior to the MLE method and another method.

\section{Application}

The numerical results of the parameters estimation and reliability estimation of PL distribution of real data have been presented. Also, we present the numerical results of the stress-strength model of PL distribution.

Firstly, we discuss an application of PL distribution using real data set to illustrate any estimation methods of PL distribution provides significant improvements over. The economic data set, which consists of 31 observations [1980:2010] on response variable GDP growth of Egypt. This data introduced by Almetwally and Almongy (2019b) and discussed by El-Sherpieny et al. (2020). The data are $(10.01132,3.756100,9.907171,7.401136,6.091518,6.602036,2.646586$, 2.519411, 7.930073, 4.972375, 5.701749, 1.078837, 4.431994, 2.900787, 3.973172, 4.642467, 4.988731, 5.491124, 4.036373, 6.105463, 5.367998, 3.535252, 2.370460, 3.192285, 4.089940, 4.478960, 6.853908, 7.090271, 7.157617, 4.673845, 5.145106).

From Table 6, it is clear that the p-values for all methods of PL distribution fit the data, since P-value $>0.05$ and Kolmogorov-Smirnov (K-S) is low. Because of the high standard error (St. $\mathrm{E})$, we used the coefficient of variation (CV) to express the precision of estimates, whenever the lower the CV the lower the dispersion. The Bayesian method estimation is the best estimation method by looking at the St. E. Although the St. E for some estimations (especially for $\hat{\lambda}$ ) are very high, but this is not a problem, because the value of the CV is low. Since the CV is more important than the St. E.

Secondly, we discuss an stress-strength reliability of PL distribution using real data set to illustrate any estimation methods of PL based on stress-strength reliability model provides significant improvements over. The real data sets of the waiting times before service of the customers of two banks A and B, respectively have been used. These data sets have been discussed by Singh et al. (2014) for estimating the stress-strength reliability in case of the Generalized Lindley distribution. The data of Bank $\mathrm{A}$ are $(0.8,0.8,1.3,1.5,1.8,1.9,1.9,2.1,2.6,2.7,2.9,3.1$, $3.2,3.3,3.5,3.6,4.0,4.1,4.2,4.2,4.3,4.3,4.4,4.4,4.6,4.7,4.7,4.8,4.9,4.9,5.0,5.3,5.5,5.7$, $5.7,6.1,6.2,6.2,6.2,6.3,6.7,6.9,7.1,7.1,7.1,7.1,7.4,7.6,7.7,8.0,8.2,8.6,8.6,8.6,8.8,8.8$, $8.9,8.9,9.5,9.6,9.7,9.8,10.7,10.9,11.0,11.0,11.1,11.2,11.2,11.5,11.9,12.4,12.5,12.9,13.0$, $13.1,13.3,13.6,13.7,13.9,14.1,15.4,15.4,17.3,17.3,18.1,18.2,18.4,18.9,19.0,19.9,20.6$, $21.3,21.4,21.9,23.0,27.0,31.6,33.1,38.5)$

The data of Bank B are $(0.1,0.2,0.3,0.7,0.9,1.1,1.2,1.8,1.9,2.0,2.2,2.3,2.3,2.3,2.5$, $2.6,2.7,2.7,2.9,3.1,3.1,3.2,3.4,3.4,3.5,3.9,4.0,4.2,4.5,4.7,5.3,5.6,5.6,6.2,6.3,6.6,6.8$, $7.3,7.5,7.7,7.7,8.0,8.0,8.5,8.5,8.7,9.5,10.7,10.9,11.0,12.1,12.3,12.8,12.9,13.2,13.7,14.5$, $16.0,16.5,28.0)$.

From Table 7, we can note the reliability in Linex case is the best estimation as it has the greatest value of reliability $(\hat{R})$ compared to other estimation methods. Also, we cannot use MPS because there are equal values in the data used. Despite the effectiveness of the MPS method in the estimate, but this problem hinders their use in the estimation process. 
Table 6: Estimate, standard Error, Coefficient of variation and Kolmogorov-Smirnov test for PL distribution.

\begin{tabular}{|c|c|c|c|c|c|c|}
\hline & & Estimate & Standard Error & $\mathrm{CV}$ & $\mathrm{K}-\mathrm{S}$ & P-Value \\
\hline \multirow[t]{3}{*}{ MLE } & $\hat{\alpha}$ & 2.12 & 1.53 & 0.72 & 0.12 & 0.73 \\
\hline & $\hat{\beta}$ & 2.84 & 0.53 & 0.19 & & \\
\hline & $\hat{\lambda}$ & 200.96 & 115.13 & 0.57 & & \\
\hline \multirow[t]{3}{*}{$\mathrm{LS}$} & $\hat{\alpha}$ & 0.68 & 1.48 & 2.16 & 0.18 & 0.22 \\
\hline & $\hat{\beta}$ & 2.47 & 2.21 & 0.90 & & \\
\hline & $\hat{\lambda}$ & 24.80 & 45.74 & 1.84 & & \\
\hline \multirow[t]{3}{*}{ MPS } & $\hat{\alpha}$ & 1.95 & 1.46 & 0.75 & 0.12 & 0.71 \\
\hline & $\hat{\beta}$ & 2.73 & 0.55 & 0.20 & & \\
\hline & $\hat{\lambda}$ & 155.33 & 91.22 & 0.59 & & \\
\hline \multirow[t]{3}{*}{ WLS } & $\hat{\alpha}$ & 1.87 & 0.53 & 0.29 & 0.10 & 0.88 \\
\hline & $\hat{\beta}$ & 2.76 & 0.20 & 0.07 & & \\
\hline & $\hat{\lambda}$ & 167.24 & 27.62 & 0.17 & & \\
\hline \multirow[t]{3}{*}{ CVM } & $\hat{\alpha}$ & 0.77 & 1.52 & 1.96 & 0.17 & 0.27 \\
\hline & $\hat{\beta}$ & 2.45 & 1.96 & 0.80 & & \\
\hline & $\hat{\lambda}$ & 29.92 & 55.57 & 1.86 & & \\
\hline \multirow[t]{3}{*}{$\mathrm{SE}$} & $\hat{\alpha}$ & 2.00 & 0.12 & 0.06 & 0.11 & 0.85 \\
\hline & $\hat{\beta}$ & 2.85 & 0.16 & 0.06 & & \\
\hline & $\hat{\lambda}$ & 200.66 & 0.30 & 0.00 & & \\
\hline \multirow[t]{3}{*}{ Linex 0.75} & $\hat{\alpha}$ & 2.00 & 0.13 & 0.06 & 0.11 & 0.81 \\
\hline & $\hat{\beta}$ & 2.86 & 0.15 & 0.05 & & \\
\hline & $\hat{\lambda}$ & 200.69 & 0.29 & 0.00 & & \\
\hline \multirow[t]{2}{*}{ Linex 1.25} & $\hat{\beta}$ & 2.87 & 0.17 & 0.06 & & \\
\hline & $\hat{\lambda}$ & 200.71 & 0.30 & 0.00 & & \\
\hline
\end{tabular}

Table 7: Estimate, standard Error and reliability of stress-strength model for PL distribution of Banks data.

\begin{tabular}{lccccccc}
\hline & $\hat{\alpha}_{1}$ & $\hat{\beta}_{1}$ & $\hat{\lambda}_{1}$ & $\hat{\alpha}_{2}$ & $\hat{\beta}_{2}$ & $\hat{\lambda}_{2}$ & $\hat{R}$ \\
\hline \multirow{2}{*}{ MLE } & 2.412786 & 1.79403 & 125.3098 & 7.34598 & 1.30233 & 79.62516 & \multirow{2}{*}{0.66544} \\
& $(1.4158)$ & $(0.24342)$ & $(55.7907)$ & $(6.5816)$ & $(0.17112)$ & $(75.7903)$ & \\
\multirow{2}{*}{ SE } & 0.732189 & 2.85345 & 266.3851 & 6.73214 & 1.3517 & 80.76017 & 0.67394 \\
& $(0.2967)$ & $(0.38624)$ & $(44.3685)$ & $(2.0186)$ & $(0.14458)$ & $(27.1558)$ & \\
\multirow{2}{*}{ Linex } & 0.75681 & 2.91363 & 264.3951 & 6.3942 & 1.2973 & 81.02873 & 0.68951 \\
& $(0.20973)$ & $(0.37916)$ & $(42.1565)$ & $(2.2963)$ & $(0.15937)$ & $(27.9171)$ & \\
\hline
\end{tabular}




\section{Conclusion}

In this paper, parameters estimation for the PL distribution are discussed based on the classical methods and the Bayesian methods. Classical methods are maximum likelihood, maximum product spacing, Ordinary Least Squares, Weighted Least Squares and Cramér-von Mises. In parameter estimation, the estimators based on MPS method behave quite better than the estimators of the classical methods, but the Bayesian estimation is the best one. In Bayesian estimation, the estimators based on LINEX loss function behave quite better than the estimators of the SE loss function, where the MSE is less than from the other methods. In reliability estimation of stress-strength model, we note that when parameters value of stress are small, the estimated model efficiency increases. By checking the previous results, where we note that MPS is better than MLE. We can conclude that the MPS method is a good alternative method to the usual MLE method in many situation, but the Bayesian estimation is the best one in reliability estimation of stress-strength model. Finally, we hope that the finding in this paper will be useful for researchers and statistician.

\section{References}

AHmad HH, Almetwally E (2020). Marshall-Olkin generalized Pareto distribution: Bayesian and non Bayesian estimation. Pakistan Journal of Statistics and Operation Research, 16(1): $21-33$.

Almetwally EM, Almongy HM (2019a). Estimation methods for the new Weibull-Pareto distribution: Simulation and application. Journal of Data Science, 17(3): 610-630.

Almetwally EM, Almongy HM (2019b). Maximum product spacing and Bayesian method for parameter estimation for generalized power Weibull distribution under censoring scheme. Journal of Data Science, 17(2): 407-444.

Almetwally EM, Almongy HM, El sayed Mubarak A (2018). Bayesian and maximum likelihood estimation for the Weibull generalized exponential distribution parameters using progressive censoring schemes. Pakistan Journal of Statistics and Operation Research, 14(4): 853-868.

Almetwaly EM, Almongy HM (2018). Bayesian estimation of the generalized power Weibull distribution parameters based on progressive censoring schemes. International Journal of Mathematical Archive, 9(6): 1-8.

Birnbaum ZW (1956). On a use of the Mann-Whitney statistic. In: Proceedings of the Third Berkeley Symposium on Mathematical Statistics and Probability, Volume 1: Contributions to the Theory of Statistics, 13-17. The Regents of the University of California.

Cheng RCH, Amin NAK (1983). Estimating parameters in continuous univariate distributions with a shifted origin. Journal of the Royal Statistical Society: Series B (Methodological), 45(3): 394-403.

Ekström M (2014). Maximum product of spacings estimation. Wiley StatsRef: Statistics Reference Online, https://doi.org/10.1002/9781118445112.stat01584.

El-Sherpieny ESA, Almetwally EM, Muhammed HZ (2020). Progressive type-II hybrid censored schemes based on maximum product spacing with application to power Lomax distribution. Physica A: Statistical Mechanics and Its Applications, 553(124657): 1-10.

Kumar D, Dey S, Nadarajah S (2017). Extended exponential distribution based on order statistics. Communications in Statistics-Theory and Methods, 46(18): 9166-9184. 
Metropolis N, Rosenbluth AW, Rosenbluth MN, Teller AH, Teller E (1953). Equation of state calculations by fast computing machines. The Journal of Chemical Physics, 21(6): 1087-1092.

Mokhlis NA, Ibrahim EJ, Gharieb DM (2017). Stress-strength reliability with general form distributions. Communications in Statistics-Theory and Methods, 46(3): 1230-1246.

Rady EHA, Hassanein WA, Elhaddad TA (2016). The power Lomax distribution with an application to bladder cancer data. SpringerPlus, 5(1): 1838.

Saraçoğlu B, Kinaci I, Kundu D (2012). On estimation of $R=P(Y<X)$ for exponential distribution under progressive type-II censoring. Journal of Statistical Computation and Simulation, 82(5): 729-744.

Singh SK, Singh U, Sharma VK (2014). Estimation on system reliability in generalized Lindley stress-strength model. Journal of Statistics Applications \& Probability, 3(1): 61-75.

Swain JJ, Venkatraman S, Wilson JR (1988). Least-squares estimation of distribution functions in Johnson's translation system. Journal of Statistical Computation and Simulation, 29(4): 271-297.

Varian HR (1975). A Bayesian approach to real estate assessment. In: Studies in Bayesian Econometric and Statistics in Honor of Leonard J. Savage, 195-208. North Holland Publishing Company.

Zellner A (1986). Bayesian estimation and prediction using asymmetric loss functions. Journal of the American Statistical Association, 81(394): 446-451. 